\title{
Integration of GaAs-based VCSEL array on SiN platform with HCG reflectors for WDM applications
}

\author{
Sulakshna Kumari ${ }^{\mathrm{a}, \mathrm{b}}$, Johan S. Gustavsson ${ }^{\mathrm{c}}$, Ruijun Wang ${ }^{\mathrm{a}, \mathrm{b}}$, Emanuel P. Haglund ${ }^{\mathrm{c}}$, Petter \\ Westbergh $^{\mathrm{c}}$, Dorian Sanchez ${ }^{\mathrm{a}, \mathrm{b}}$, Erik Haglund ${ }^{\mathrm{c}}$, Åsa Haglund ${ }^{\mathrm{c}}$, Jörgen Bengtsson ${ }^{\mathrm{c}}$, Nicolas Le \\ Thomas $^{\mathrm{a}, \mathrm{b}}$, Gunther Roelkens ${ }^{\mathrm{a}, \mathrm{b}}$, Anders Larsson ${ }^{\mathrm{c}}$, and Roel Baets ${ }^{\mathrm{a}, \mathrm{b}}$ \\ ${ }^{a}$ Photonics Research Group, INTEC Department, Ghent University-imec, Belgium, \\ ${ }^{\mathrm{b}}$ Center for Nano- and Biophotonics, Ghent University, Belgium, \\ 'Photonics Laboratory, Department of Microtechnology and Nanoscience, Chalmers University of \\ Technology, Gothenburg, Sweden
}

\begin{abstract}
We present a GaAs-based VCSEL structure, BCB bonded to a $\mathrm{Si}_{3} \mathrm{~N}_{4}$ waveguide circuit, where one DBR is substituted by a free-standing $\mathrm{Si}_{3} \mathrm{~N}_{4}$ high-contrast-grating (HCG) reflector realized in the $\mathrm{Si}_{3} \mathrm{~N}_{4}$ waveguide layer. This design enables solutions for on-chip spectroscopic sensing, and the dense integration of 850-nm WDM data communication transmitters where individual channel wavelengths are set by varying the HCG parameters. RCWA shows that a 300nm-thick $\mathrm{Si}_{3} \mathrm{~N}_{4}$ HCG with $800 \mathrm{~nm}$ period and $40 \%$ duty cycle reflects strongly (>99\%) over a $75 \mathrm{~nm}$ wavelength range around $850 \mathrm{~nm}$. A design with a standing-optical-field minimum at the III-V/airgap interface maximizes the HCG's influence on the VCSEL wavelength, allowing for a 15-nm-wide wavelength setting range with low threshold gain $\left(<1000 \mathrm{~cm}^{-1}\right)$.
\end{abstract}

Keywords: silicon photonics, silicon nitride waveguide, vertical cavity surface emitting laser, integration, high contrast grating

\section{INTRODUCTION}

Si photonics technology is rapidly gaining attention in various fields due to its high index contrast and compatibility with standard complementary metal-oxide semiconductor (CMOS) technology. However, due to the absorption in silicon below a wavelength of $1.1 \mu \mathrm{m}$, silicon is not suitable for waveguide circuits in the visible or near-infrared (VIS-NIR). In recent years silicon nitride $\left(\mathrm{Si}_{3} \mathrm{~N}_{4}\right)$ is emerging as a promising material platform for such applications due to its VIS-NIR transparency, compatibility with CMOS fabrication technology, tight confinement and lower temperature sensitivity compared to silicon [1]. For spectroscopic applications the VIS-NIR (500-950 nm) wavelength window is of interest due to minimal photodamage to living cells, negligible water absorption, large Raman scattering cross-section etc. Also to meet the demands of optical interconnects in future datacom applications, there is a need for dense integration of lowcost, high-bandwidth, and power-efficient WDM transmitters using photonic integrated circuits (PICs). However, the realization of CMOS compatible light sources is considered to be the biggest challenge in the silicon photonics world. Even though silicon can route light very efficiently it cannot be used as a light emitter since it has an indirect bandgap. An attractive approach is to use hybrid solutions, in which III-V semiconductor materials are either transferred by bonding or heteroepitaxially grown on silicon. There are different techniques to transfer III-V material such as direct bonding and adhesive bonding, each with its own advantages and disadvantages [2]. Recent developments in adhesive bonding show that a very reliable homogeneous ultrathin benzocyclobutene (BCB) bonding is possible, making it attractive as it is tolerant to surface roughness, defects and contamination of the semiconductor surfaces [3]. An attractive light source in III-V materials is the GaAs-based vertical-cavity surface-emitting laser (VCSEL), which has several advantages compared to edge-emitting lasers such as on-wafer level testing, low threshold current and efficient high-speed modulation at low currents. Recently a high-reflectivity broadband mirror constituting of a high-indexcontrast subwavelength grating (HCG) has been proposed to replace the traditional DBR structure in VCSELs [4, 5,]. The origin of the ultra-broadband high reflectance in such a grating is explained to be a destructive interference effect between two grating modes [6]. These HCG mirrors can provide several advantages over DBRs such as substantial thickness reduction, high polarization sensitivity. In addition, the use of a HCG in a resonator configuration, can offer

High Contrast Metastructures IV, edited by Connie J. Chang-Hasnain, David Fattal,

Fumio Koyama, Weimin Zhou, Proc. of SPIE Vol. 9372, 93720U · C 2015 SPIE

CCC code: $0277-786 \mathrm{X} / 15 / \$ 18 \cdot$ doi: $10.1117 / 12.2077090$

Proc. of SPIE Vol. $937293720 \mathrm{U}-1$ 
suppression of higher order transverse modes and the post-epitaxial growth resonator wavelength setting by changing the grating parameters $[4,5]$.

The basic idea here is to realize an integrated VCSEL in which a half GaAs VCSEL is integrated on a $\mathrm{Si}_{3} \mathrm{~N}_{4}$ HCG mirror using polymer adhesive bonding using BCB [7], thereby substituting one of the distributed Bragg reflectors by a free-standing $\mathrm{Si}_{3} \mathrm{~N}_{4}$ high-contrast-grating (HCG) reflector made in the $\mathrm{Si}_{3} \mathrm{~N}_{4}$ waveguide layer.

\section{HIGH CONTRAST GRATING DESIGN}

High contrast gratings (HCG) are structures with a period less than but near to the optical design wavelength, and a high refractive index contrast between the grating bars and the surrounding medium. They have many interesting properties such as very broadband high reflectance and narrow high Q factor resonances [8]. In recent years, HCGs with broadband high reflectivity have been reported to replace top DBRs of VCSELs and tuning of the lasing wavelength have also been achieved by electrostatic actuation of the HCG [4]. Typically, HCGs are realized in high index materials such as Si and $\mathrm{GaAs}$, and it is still to be explored if $\mathrm{Si}_{3} \mathrm{~N}_{4}$, which has a lower refractive index ( $\approx 2$ @ 850nm), can yield HCGs with similar performance. Figure 1 shows the cross-sectional schematic view of an HCG and the definitions of the grating parameters.

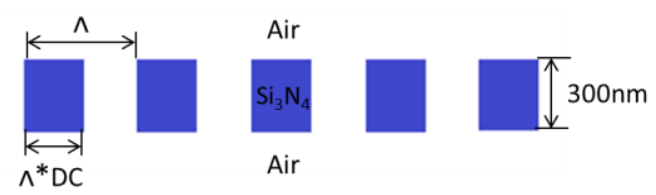

Figure 1. Cross-sectional schematic view of an HCG.

To efficiently simulate the diffraction of electromagnetic waves by a periodic structure such as the HCG, Rigorous Coupled-Wave Analysis (RCWA) can be used [9]. We used the in-house developed RCWA software named RODIS [10]. Optimization of the grating parameters are performed by computing the maximum variation range of the grating parameters for which the HCG reflects $>99 \%$ for TE polarization at $850 \mathrm{~nm}$. These grating parameters are the period of the grating $(\Lambda)$, the duty cycle ( $\mathrm{DC}=$ width of high index material/period of grating), and the thickness of the silicon nitride layer. The optimized grating parameters are chosen such that a maximum tolerance on these parameters is obtained, while at the same time giving high reflection bandwidth. The optimized parameters are a grating period $(\Lambda)$ of $800 \mathrm{~nm}$, a duty cycle of 0.40 and a thickness of the nitride layer of $300 \mathrm{~nm}$ giving a reflection bandwidth of $75 \mathrm{~nm}(>99 \%$ reflectivity) centered at $850 \mathrm{~nm}$, yielding a $9 \%$ stopband-to-center-wavelength ratio as shown in Figure 2 [7]. The high reflectivity band arises due to the presence of two leaky grating modes shown as two transmission dips inside the high reflection band in Figure 2. These transmission dips correspond to a guided mode resonance at which the transmittance approaches zero [11].

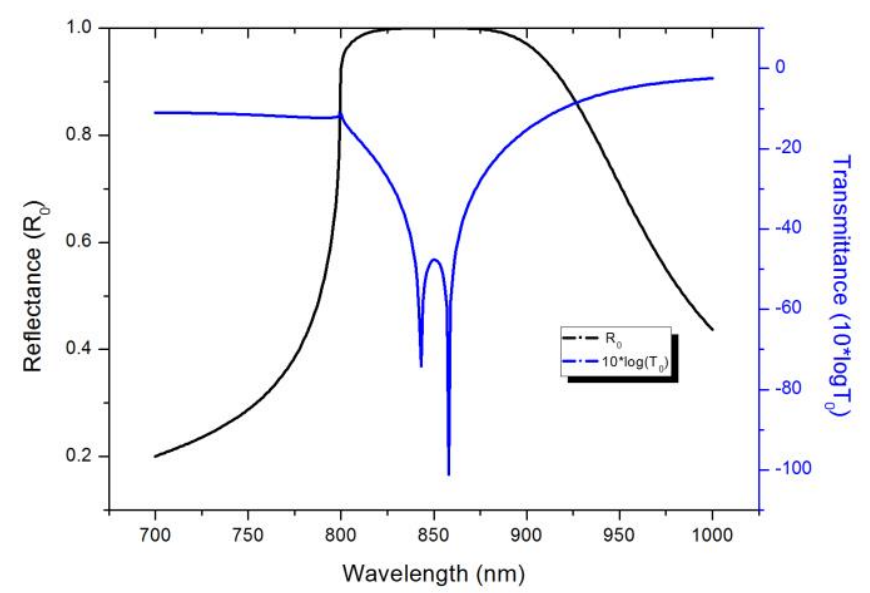

Figure 2. Reflectivity (black curve) and transmission on a logarithmic (blue curve) scale as a function of wavelength for TE polarization for a grating with the following parameters: period $800 \mathrm{~nm}, \mathrm{DC}=40 \%, \mathrm{Si}_{3} \mathrm{~N}_{4}$ thickness $300 \mathrm{~nm}$. 


\section{VCSEL DESIGN}

We present a novel design of a GaAs-based VCSEL, bonded to a $\mathrm{Si}_{3} \mathrm{~N}_{4}$ waveguide platform using $\mathrm{BCB}$, where one of the distributed Bragg reflectors is completely substituted by a free-standing $\mathrm{Si}_{3} \mathrm{~N}_{4}$ HCG reflector made in the $\mathrm{Si}_{3} \mathrm{~N}_{4}$ waveguide layer. A schematic of the VCSEL design is shown in Figure 3. The device consists of a GaAs-based half VCSEL (one DBR + active region) that is positioned on top of a free standing $\mathrm{Si}_{3} \mathrm{~N}_{4}$ grating acting as the bottom mirror. The half VCSEL has a p-doped DBR (24 mirror pairs of $\mathrm{Al}_{0.12} \mathrm{Ga}_{0.88} \mathrm{As}_{\mathrm{Al}} \mathrm{Al}_{0.9} \mathrm{Ga}_{0.1} \mathrm{As}$ ), an active region (five $\mathrm{Al}_{0.37} \mathrm{GaAs} / \mathrm{In}_{0.1} \mathrm{GaAs}$ quantum wells), and an $\mathrm{n}$-doped current spreading layer for intra-cavity contacting. For lateral current and optical confinement an oxide aperture is located in the p-doped DBR just above the active region.

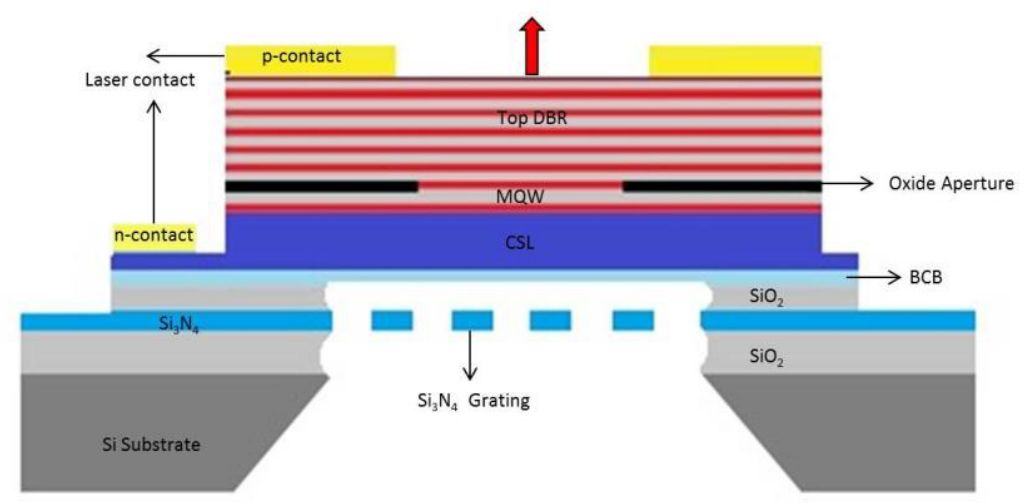

Figure 3. Schematic cross-sectional view of the VCSEL, consisting of a half VCSEL on top of a free-standing $\mathrm{Si}_{3} \mathrm{~N}_{4}$ grating.

\subsection{Principle of wavelength setting}

By altering the grating parameters of the HCG both the reflectivity spectrum as well as the phase of the reflection can be changed, as exemplified in Figure 4. The VCSEL emission wavelength depends on the round trip phase condition of the laser cavity. Hence by slightly changing the grating period or duty cycle, the VCSEL emission wavelength can be altered, allowing the realization of VCSELs with different emission wavelengths within one VCSEL array chip.
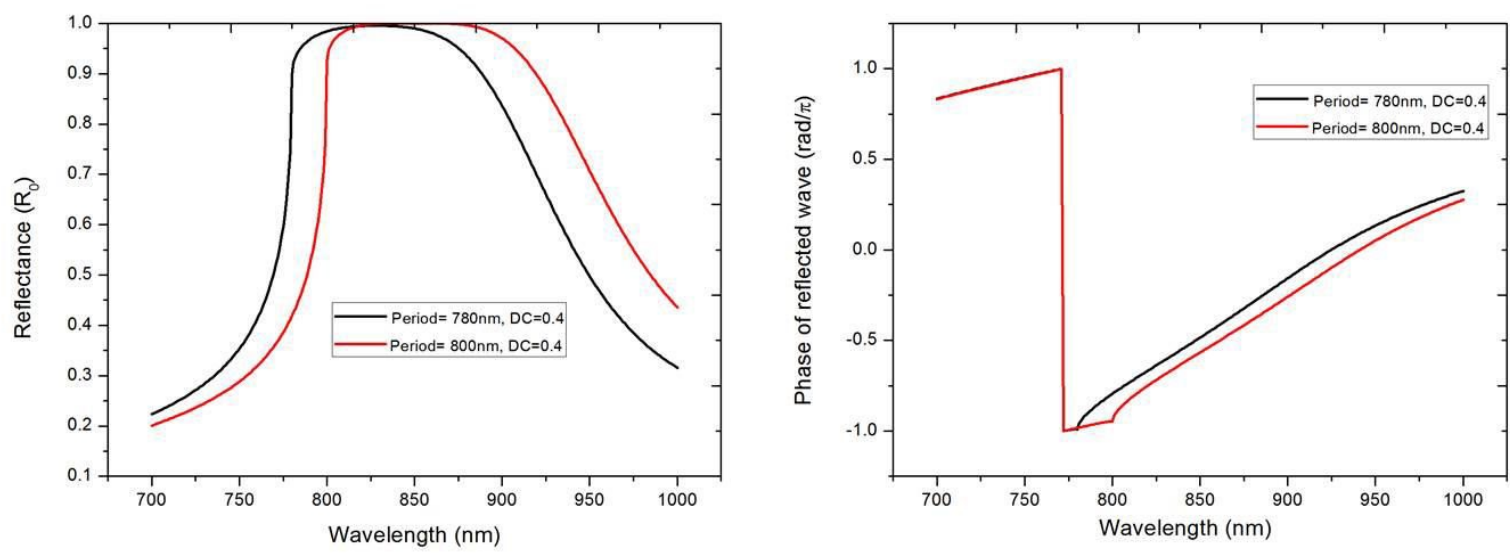

Figure 4. (a) Power reflectance and (b) phase of the reflected wave for two different grating periods. 


\subsection{Wavelength setting range}

To theoretically investigate the possible wavelength setting range, a 1D-wave transfer matrix method is applied to simulate the detailed VCSEL epitaxial layer structure, where the HCG is replaced by an "artificial" interface having the spectral reflectance/transmittance properties obtained from the RCWA calculations. All other important cavity properties (longitudinal confinement factor, absorption loss, top and bottom mirror loss) are extracted. We compare the VCSEL wavelength setting performance of a design where the internal optical field standing-wave has a maximum at the IIIV/airgap interface to one where the standing wave is minimum at the same interface. In the following we refer to those two cases as resonant and non-resonant half-VCSEL design, where the only physical difference is a one quarterwavelength thick layer.

An anti-reflection coating (ARC) using a low refractive index material such as SiON with proper thickness at the III-V/airgap interface can increase the wavelength setting range of VCSELs [12]. However, the deposition of an extra ARC layer on the III-V epitaxy makes the fabrication of the whole device complicated, as selective etching of $\mathrm{SiO}_{2}$ to the ARC is difficult. An extra thickness of $\lambda / 4 \mathrm{n}$ in the n-doped current spreading layer of the resonant half-VCSEL design, yields a non-resonant half-VCSEL design, and has a similar effect on the wavelength tuning range as adding an ARC. By having a minimum in the optical field standing-wave at the III-V/airgap interface the optical reflection at this boundary is minimized and the bottom mirror reflectivity will mainly be determined by the HCG, giving the HCG a bigger influence on setting the resonance wavelength which should in turn also yield a larger wavelength setting range. Figure 5 shows numerical results for the resonant half-VCSEL design case. As can be seen in Figure 5(left) for a grating with period between 780 and $840 \mathrm{~nm}$ and a duty cycle between $33 \%$ and $47 \%$, the threshold gain is below $1000 \mathrm{~cm}^{-1}$, which is typically required for achieving acceptable VCSEL output performance. The resonance wavelength for those grating parameters ranges only 4nm, see Figure 5(right). For the corresponding non-resonant half-VCSEL design case, shown in Figure 6, the possible wavelength setting range is $15 \mathrm{~nm}$, even though for a smaller duty cycle range. We clearly find that a design with a standing-wave minimum at the III-V/airgap interface will maximize the HCG's influence on determining the VCSEL resonance wavelength, and thereby allowing for a 15 -nm-wide wavelength setting range with low threshold gain $\left(<1000 \mathrm{~cm}^{-1}\right)$.
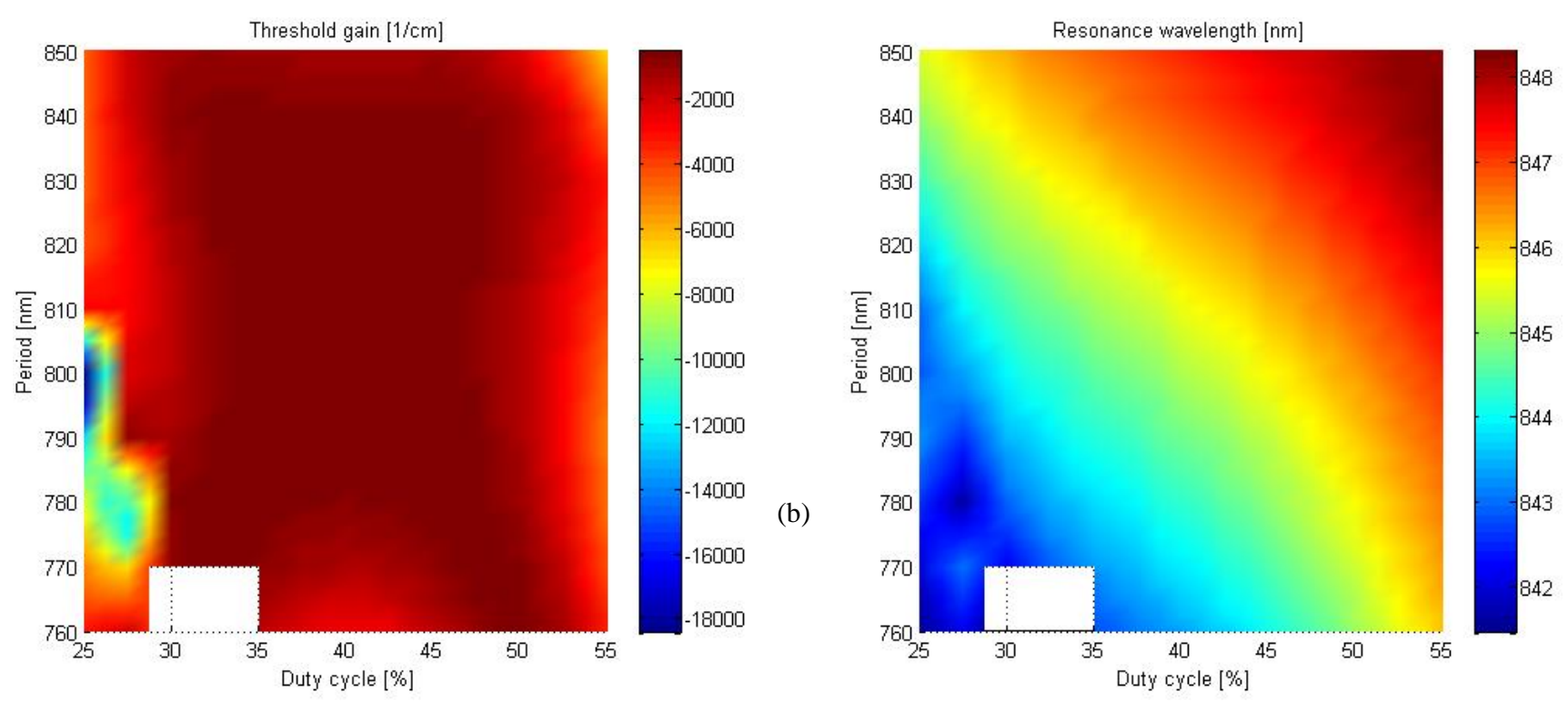

Figure 5. Resonant half-VCSEL design case. (left) Threshold gain as function of grating period and DC, and (right) gresonance wavelength as function of grating period and DC. 

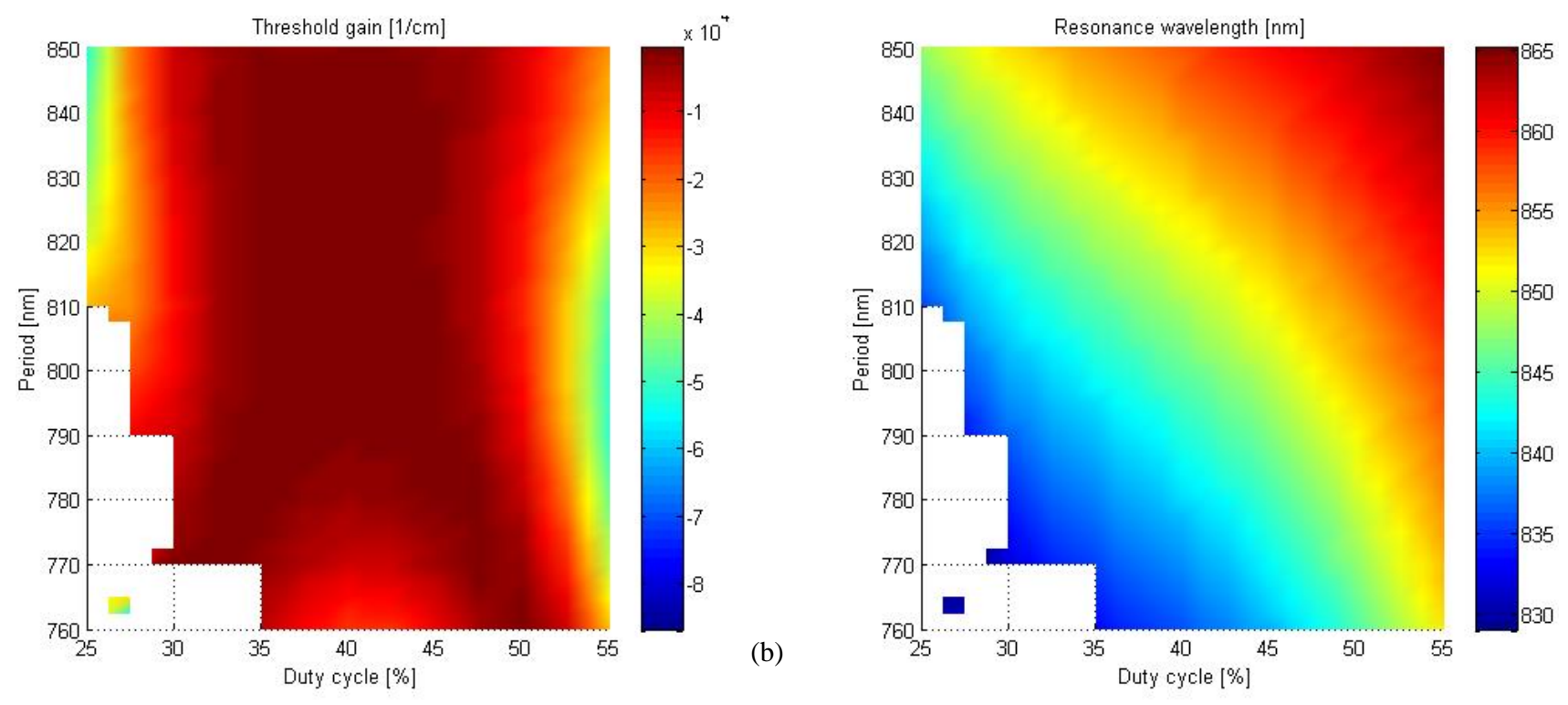

Figure 6. Non-resonant half-VCSEL design case. (left) Threshold gain as function of grating period and DC, and (right) resonance wavelength as function of grating period and DC.

\section{GRATING FABRICATION AND EVALUATION}

In the full VCSEL process, the idea is to use adhesive BCB bonding to join the GaAs-based half VCSEL epitaxial material to the already fabricated $\mathrm{Si}_{3} \mathrm{~N}_{4}$ HCG. To facilitate the bonding the HCG has to be planarized with $\mathrm{SiO}_{2}$ before the bonding, and thus the bonding interface will be between $\mathrm{BCB}$ and $\mathrm{SiO}_{2}$. After the bonding, the GaAs substrate is completely removed by mechanical grinding and a wet etch process. The VCSELs will then be fabricated in a standard VCSEL process, followed by a release of the HCG from the backside of the Si wafer using KOH to etch the Si substrate and buffered hydrofluoric acid (BHF) to etch the $\mathrm{SiO}_{2}$ which surrounds the $\mathrm{Si}_{3} \mathrm{~N}_{4}$ grating beams.

So far, we have focused on the fabrication of the $\mathrm{Si}_{3} \mathrm{~N}_{4} \mathrm{HCGs}$ and tested the release from the backside of the $\mathrm{Si}$ wafer. For a successful fabrication, the $\mathrm{Si}_{3} \mathrm{~N}_{4}$ must have a low built-in stress and at the same time have a large etch persistence to BHF compared to $\mathrm{SiO}_{2}$. The $\mathrm{Si}_{3} \mathrm{~N}_{4}$ can be deposited using either plasma-enhanced chemical vapor deposition (PECVD) or low-pressure chemical vapor deposition (LPCVD). PECVD deposited $\mathrm{Si}_{3} \mathrm{~N}_{4}$ offers films with lower stress, but with lower wet etch selectivity to $\mathrm{SiO}_{2}$ in BHF. LPCVD $\mathrm{Si}_{3} \mathrm{~N}_{4}$ on the other hand provides good wet etch selectivity to $\mathrm{SiO}_{2}$, an excellent control of the homogeneity of material index and thickness, but a high built-in stress, particularly in thicker films (>300 nm), due to the high temperature deposition process (>700C) [1]. Due to the higher wet etch selectivity we have chosen to work with LPCVD $\mathrm{Si}_{3} \mathrm{~N}_{4}$.

The grating fabrication process is done at IMEC and starts with the deposition of a $1.6 \mu \mathrm{m}$ thick $\mathrm{SiO} 2$ layer by PECVD followed by a 300nm thick LPCVD $\mathrm{Si}_{3} \mathrm{~N}_{4}$ layer on 200mm Si wafers. The gratings are then defined by $193 \mathrm{~nm}$ optical lithography, etched by inductive coupled fluorine-based etching [1] and planarized by PECVD deposited $\mathrm{SiO}_{2}$. The release of the grating beams is then done from the backside of the silicon wafer by KOH etching of the Si and BHF etching of the $\mathrm{SiO}_{2}$. Critical point drying in a Leica EM CPD300 is used to avoid sticktion and collapse of the grating beams. Figure 7 shows the microscopic and SEM images of the fabricated free standing gratings. The grating beams show no bending, but have a slightly trapezoidal cross-section, which to some extent will degrade the grating's reflectivity characteristic. 


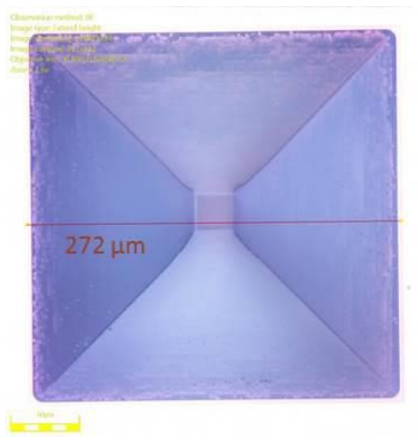

(a)

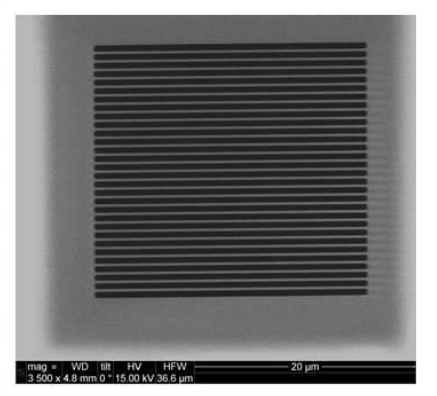

(b)

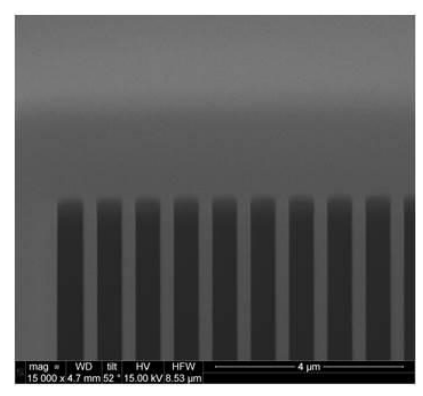

(c)

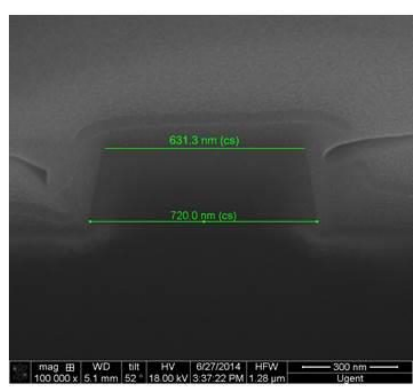

(d)

Figure 7. Fabricated free standing $\mathrm{Si}_{3} \mathrm{~N}_{4}$ grating (a) Microscopic image taken from the backside of the Si wafer (b) SEM image taken from the top of the grating. (c) a $52^{\circ}$ tilted SEM view of the grating (d) cross-sectional SEM view of the $\mathrm{Si}_{3} \mathrm{~N}_{4}$ waveguide showing the topology of the grating bars.

Reflection spectra of the fabricated gratings were measured using a set-up consisting of a fiber coupled supercontinuum light source, a fiber collimator, a beamsplitter, a microscope objective $(\mathrm{NA}=0.8)$ and an output fiber connected to an optical spectrum analyser. The measured reflection spectrum from the grating is normalized to that of a calibrated gold mirror (Thorlabs). A comparison between the experimentally measured reflectance spectrum and that obtained from FDTD simulations of a finite grating of 30 periods are shown in Figure 8. The absolute reflectance values do not match completely, but the spectral characteristics are in good agreement.

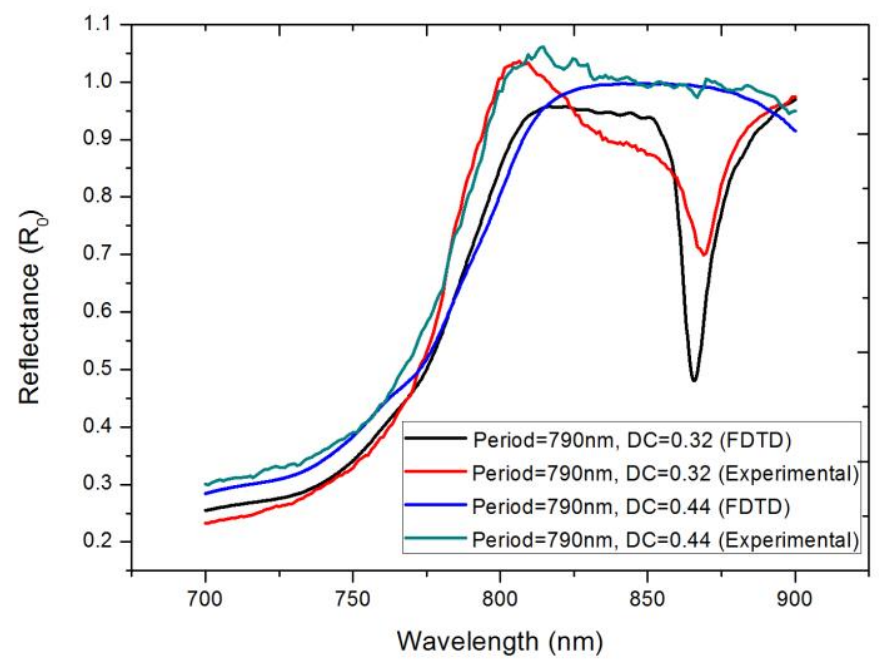

Figure 8. Measured and simulated reflection spectra from a $\mathrm{Si}_{3} \mathrm{~N}_{4}$-based HCG.

\section{CONCLUSIONS}

We present a novel design of a half GaAs-based VCSEL, bonded to a $\mathrm{Si}_{3} \mathrm{~N}_{4}$ waveguide platform using BCB, where one of the DBRs is completely substituted by a free-standing $\mathrm{Si}_{3} \mathrm{~N}_{4}$ HCG. The wavelength setting properties are explored numerically where the use of a resonant and non-resonant GaAs-based half-VCSEL designs are compared. We show that a non-resonant design maximizes the HCG's influence on setting the VCSEL resonance wavelength, allowing for a 15nm-wide wavelength setting range with a low threshold gain $\left(<1000 \mathrm{~cm}^{-1}\right)$. The first initial results of fabricated $\mathrm{Si}_{3} \mathrm{~N}_{4}$ HCG are also presented, showing free-standing grating bars with no bending and a slight trapezoidal cross-section. Measured reflectivity spectra show good agreement with those obtained from FDTD simulations of finite gratings. 


\section{REFERENCES}

[1] A. Z. Subramanian, P. Neutens, A. Dhakal, R. Jansen, T. Claes, X. Rottenberg, F. Peyskens, S. Selvaraja, P. Helin, B. Du Bois, K. Leyssens, S. Severi, P. Deshpande, R. Baets, and P. Van Dorpe., "Low-Loss Singlemode PECVD Silicon Nitride Photonic Wire Waveguides for 532-900 nm Wavelength Window Fabricated Within a CMOS Pilot Line", IEEE Photonics Journal, 5(6), 2202809 (2013).

[2] Di Liang, Gunther Roelkens, Roel Baets and John E. Bowers., "Hybrid Integrated Platforms for Silicon Photonics", Materials 2010, 3, 1782-1802; doi:10.3390/ma3031782 (2010).

[3] S. Keyvaninia, M. Muneeb, S. Stanković, P. J. Van Veldhoven, D. Van Thourhout, and G. Roelkens., “ Ultrathin DVS-BCB adhesive bonding of III-V wafers, dies and multiple dies to a patterned silicon-on-insulator substrate", Oprtical Materials Express, 3(1), 35-46 (2013).

[4] C. F. R. Mateus, M. C. Y. Huang, D. Yunfei, A. R. Neureuther, and C. J. Chang-Hasnain., "Ultrabroadband mirror using low-index cladded subwavelength grating", IEEE Photonics Technology Letter, 16(2), 518-520, (2004).

[5] M. C. Y. Huang, Y. Zhou, and C. J. Chang-Hasnain., "A surface-emitting laser incorporating a high-index contrast subwavelength grating", Nature Photonics., Vol. 1, 119-122 (2007).

[6] Vadim Karagodsky, Forrest G. Sedgwick, and Connie J. Chang-Hasnain., "Theoretical analysis of subwavelength high contrast grating reflectors", Optics Express, 18(16), 16973-16988 (2010).

[7] S. Kumari1, R. Wang, D. Sanchez, J. Gustavsson, R. Safaisini, A. Haglund, J. Bengtsson, A. Larsson, G. Roelkens, R. Baets,. "Silicon Nitride based high contrast grating for heterogeneously integrated tunable VCSELs.", Proceedings Symposium IEEE Photonics Society Benelux, 231-234 (2013).

[8] Ye Zhou, Michael C. Y. Huang, Christopher Chase, Vadim Karagodsky, Michael Moewe, Bala Pesala, Forrest G. Sedgwick, and Connie J. Chang-Hasnain., "High-Index-Contrast Grating (HCG) and Its Applications in Optoelectronic Devices", IEEE Journal of Selected Topic in Quantum Electron. 15(5), 1485-1499 (2009).

[9] M. G. Moharam and T. K. Gaylord., "Rigorous coupled-wave analysis of planar-grating diffraction", JOSA, 71( 7), 811-818 (1981).

[10] http://photonics.intec.ugent.be/research/facilities/design/rodis/

[11] Robert Magnusson and Mehrdad Shokooh-Saremi, "Physical basis for wideband resonant reflectors", Optics Express, 16(5), 3456-3462 (2008).

[12] Thor Ansbæk, Il-Sug Chung, Elizaveta S. Semenova, and Kresten Yvind., "1060-nm Tunable Monolithic High Index Contrast Subwavelength Grating VCSEL”, 25(4), 365-367 (2013). 